\title{
OCT-Diagnostik beim Glaukom: Tipps \& Tricks
}

Christian Mardin

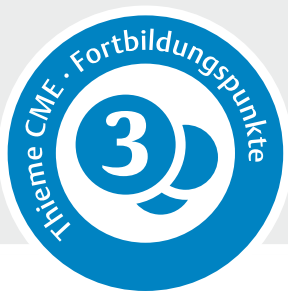

\begin{abstract}
Die Technik der optischen Kohärenztomografie scheint bei Glaukomen mittlerweile unverzichtbar für Dokumentation, Diagnosehilfestellung und Verlaufsbeobachtung. Die kritisch betrachteten Befunde sollten immer in Übereinstimmung mit dem klinischen Bild des Patienten gesehen werden. Der Artikel zeigt die Einsatzmöglichkeiten anhand von Fallbeispielen.
\end{abstract}

$\begin{array}{ll}\text { ABKÜRZUNGEN } \\ \text { AMD } & \text { altersassoziierte Makuladegeneration } \\ \text { APS } & \text { Automated Positioning System } \\ \text { BMO } & \text { Bruch-Membran-Öffnung } \\ \text { BMO-MRW minimale neuroretinale Randsaumbreite } \\ \text { GCL } & \text { Ganglienzellschicht } \\ \text { NRR } & \text { neuroretinaler Randsaum } \\ \text { OCT } & \text { optische Kohärenztomografie } \\ \text { RNF } & \text { retinale Nervenfaserschicht } \\ \text { RNFL } & \text { retinale Nervenfaserschichtdicke } \\ \text { SD-OCT } & \text { Spectral-Domain OCT } \\ \text { SS-OCT } & \text { Swept-Source OCT } \\ \text { TSNIT } & \text { temporal-superior-nasal-inferior- } \\ & \text { temporal } \\ & \\ \end{array}$

\section{Einleitung}

Die Technik der optischen Kohärenztomografie (OCT) ist in der Glaukomdiagnostik einer der großen Meilensteine geworden. Spectral-Domain OCT (SD-OCT) und SweptSource OCT (SS-OCT) ermöglichen hochauflösende und schnelle Bildaufnahmen mit bis zu 100000 A-Scans pro Sekunde. Damit sind empfindliche Strukturen wie die Ganglienzellschicht der Makula, die retinale Nervenfaserschicht und der Sehnervenkopf bis zur Lamina cribrosa mit einer digitalen axialen Auflösung von bis zu 3,9 $\mu \mathrm{m}$ ähnlich einem in vivo histologischen Schnitt darstellbar. Die Variabilität der Messungen beträgt nur 1,4 $\mu \mathrm{m}$ [1].

Die hohe Präzision der Messungen mit immer höherer Auflösung erlaubt es, den natürlichen Altersverlust von neuroretinalem Randsaum (NRR) und retinaler Nervenfaserschicht (RNFL) zu messen. Diesen von frühen glaukomatösen Veränderungen zu trennen, ist eine Herausforderung für jede Messmethode. Da jeder Patient eine individuelle Progressionsrate zeigt, sind wiederholte präzise Messungen essenziell [2].
Die hochauflösende OCT-Technik hat schnell Verbreitung in der alläglichen Versorgung gefunden. Die Glaukomfrüherkennung konnte mit der Messung der RNFL und der Ganglienzellschicht (GCL) weiter vorangebracht werden, mit hoher Sensitivität im präperimetrischen Glaukomstadium. Die Messung der makulären GCL-Dicke mit dem SD-OCT zeigt ebenfalls eine gute Diskriminierung zwischen normalen und frühen Glaukomen.

Oddone et al. [3] fanden in einer Literaturübersicht zu drei unterschiedlichen OCT-Geräten einen Vorteil der RNFL-Dickenmessung gegenüber der makulären Ganglienzellschichtdickenmessung. Die neueste Anwendung ist die Bestimmung der minimalen neuroretinalen Randsaumbreite (BMO-MRW) in der Papille unter Zuhilfenahme der Detektion des Endes der Bruch-Membran und der Membrana limitans interna als präzise darstellbare Papillengrenze [4]. Die BMO-MRW wird an 48 Punkten gemessen und zu sektoriellen oder gesamten Werten extrapoliert. Die BMO-MRW zeigte in ersten klinischen Studien eine fast ähnlich hohe Diskriminierung von Glaukomen wie die retinale Nervenfaserschichtdicke mit der OCT [4-6]. Die extrapolierte Fläche der BMO gibt einen groben Hinweis auf die Papillenfläche und korreliert nur marginal mit der mit dem HRT gemessenen Papillenfläche, die den Innenrand des Elschnig-Skleralrings als Grundlage hat.

\footnotetext{
Cave

Die Öffnung der BMO und Papillenfläche sind nicht gleichzusetzen.
}

Dieser Artikel soll zu einigen Fragen bei der Beurteilung von OCT-Darstellungen bei Glaukomen Antworten geben. 


\section{Wie stellen sich mit OCT glaukomrelevante Messungen dar?}

Die Messwerte der retinalen Nervenfaserschicht (RNFL) und der minimalen neuroretinalen Randsaumbreite (BMO-MRW) werden $360^{\circ}$ in einer gestreckten TSNITGrafik (temporal-superior-nasal-inferior-temporal) dargestellt, wobei die aktuelle Messung den Messwerten einer Normalpopulation gegenübergestellt wird. Die zweifache Standardabweichung wird in Grün, die 95\%Perzentile in Gelb (grenzwertig) und 99\%-Perzentile in Rot (pathologisch) in verschiedenen Sektoren und als Gesamtwert, gemittelt über alle Sektoren, dargestellt.

Dickenwerte der Ganglienzellschicht eines Makulavolumens werden in regionalen Absolutwerten (auch als Topografiekarte) oder ebenfalls farbkodiert im Vergleich zu Normwerten dargestellt. Abweichungen zu Normdicken erscheinen in Gelb und Rot und geben einen raschen Überblick über die Topografie der Makulaschichten im Aufblick auf den Augenhintergrund.
Eine beispielhafte Übersichtsdarstellung für ein rechtes Auge zeigt $>$ Abb. 1.

\section{Was spricht für ein Glaukom?}

Bei der OCT-Befundung sollte zunächst das Augenmerk auf die RNFL gelegt werden (bei frühen Glaukomen am aussagekräftigsten [6]), gefolgt von der minimalen neuroretinalen Randsaumbreite (BMO-MRW) und Dicke der Ganglienzellschicht (GCL). Das Höhenprofil von RNFL und BMO-MRW sollte harmonische Übergänge von Gipfel zu Tal haben. Steile Abbrüche oder Einkerbungen im Höhenprofil sprechen für fokale Defekte der RNFL oder der minimalen neuroretinalen Randsaumbreite (am häufigsten temporal unten und oben).

Das Dickenprofil sollte im grünen Referenzbereich liegen und man kommt nicht umhin, die B-Scan-Bilder und Segmentierungen sich noch einmal genau anzuschauen.

Das Infrarot-Fundusbild kann Informationen zu möglichen Papillenrandblutungen (Cave: sind aber nur in 50\% der Fälle sichtbar), zur parapapillären Atrophiezone und
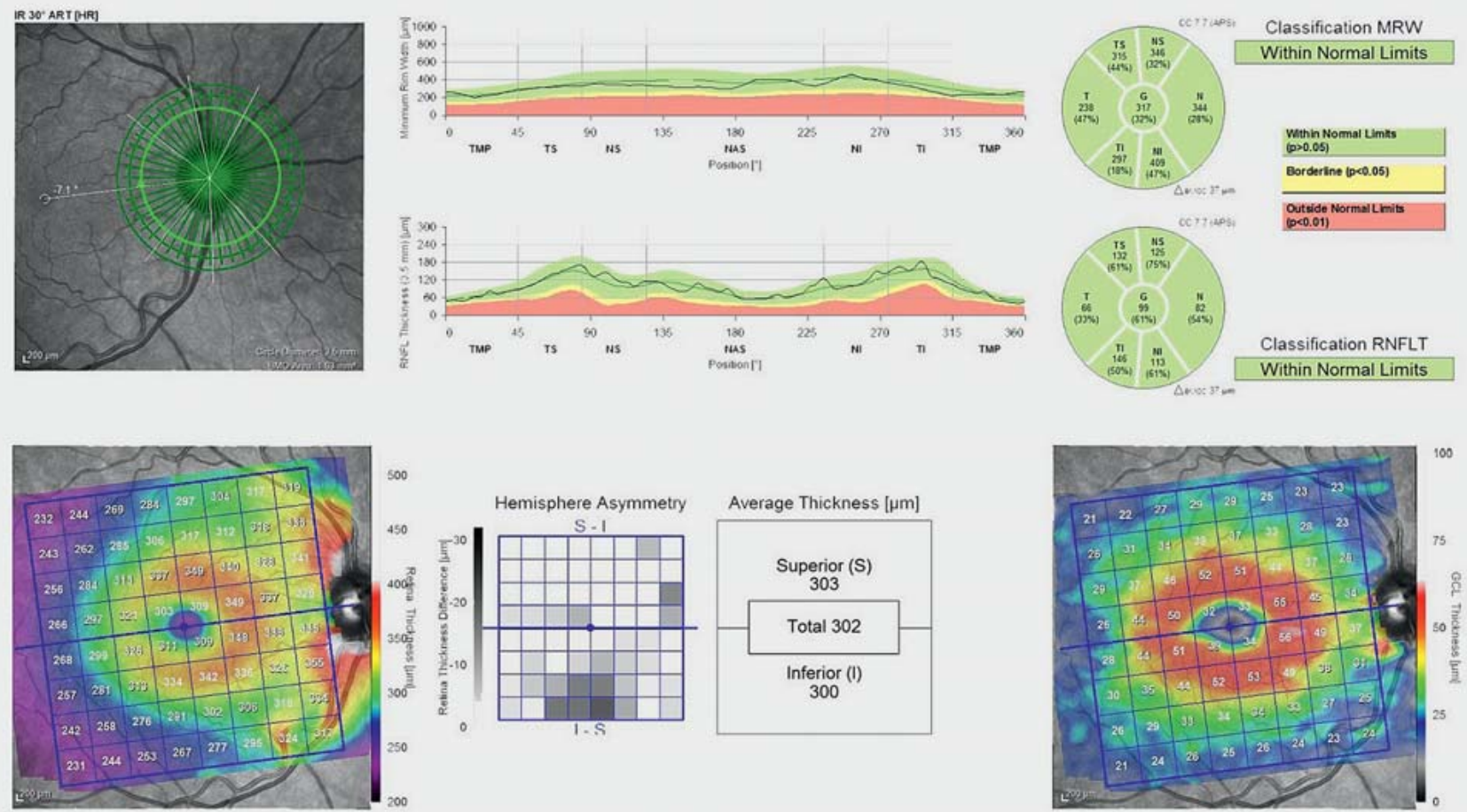

Reference database: European Descent (2014)

- Abb. 1 Beispielhaft eine Übersichtsdarstellung für ein rechtes Auge mit den TSNIT-Grafiken (temporal-superior-nasal-inferior-temporal) für minimale neuroretinale Randsaumbreite (BMO-MRW), retinale Nervenfaserschicht (RNFL) (oben) und Dicke der Netzhaut und der Ganglienzellschicht (GCL) (unten). Zwischen den Grafiken unten eine Darstellung zur hemisphärischen Makulasymmetrie (der Grad der Abweichung grau kodiert). 


\section{FALLBEISPIEL 1}

Rechtes Auge einer Patientin, 32 Jahre alt. Familiäres juveniles Offenwinkelglaukom mit bei Diagnosestellung sehr hohen intraokularen Druckwerten über $40 \mathrm{mmHg}$. Jetzt regulierter, intraokularer Druck von 9-20 mmHg. Der Fernvisus beträgt beidseits 1,0, die zentrale Hornhautdicke beträgt R $542 \mu \mathrm{m}$, L536 $\mu \mathrm{m}$. Die Papille ist leicht exkaviert (a, weißer Pfeil), blass; wahrscheinlich aufgrund der initialen hohen Drucklage kommt eine einfache Atrophiekomponente hinzu. Das Gesichtsfeld ist beginnend glaukomatös verändert (b, blaue Pfeile).

Die retinale Nervenfaserschicht (RNFL) ist aufgrund der einfachen Komponente der Optikusatrophie deutlich in der Schichtdicke verringert (d unten), die minimale neuroretinale Randsaumbreite (BMO-Fläche 2,56 mm²) vor allem im unteren Bereich der Papille atrophiert (d oben, schwarzer Pfeil). Die makuläre Ganglienzellschicht ist in der Topografie unten und temporal gemindert (c, kalte Farben, weiße Pfeile), und in der Differenzkarte zur Normdatenbank ist die Verdünnung jenseits der 99\%-Perzentile in rot dargestellt (e, weißer Pfeil).
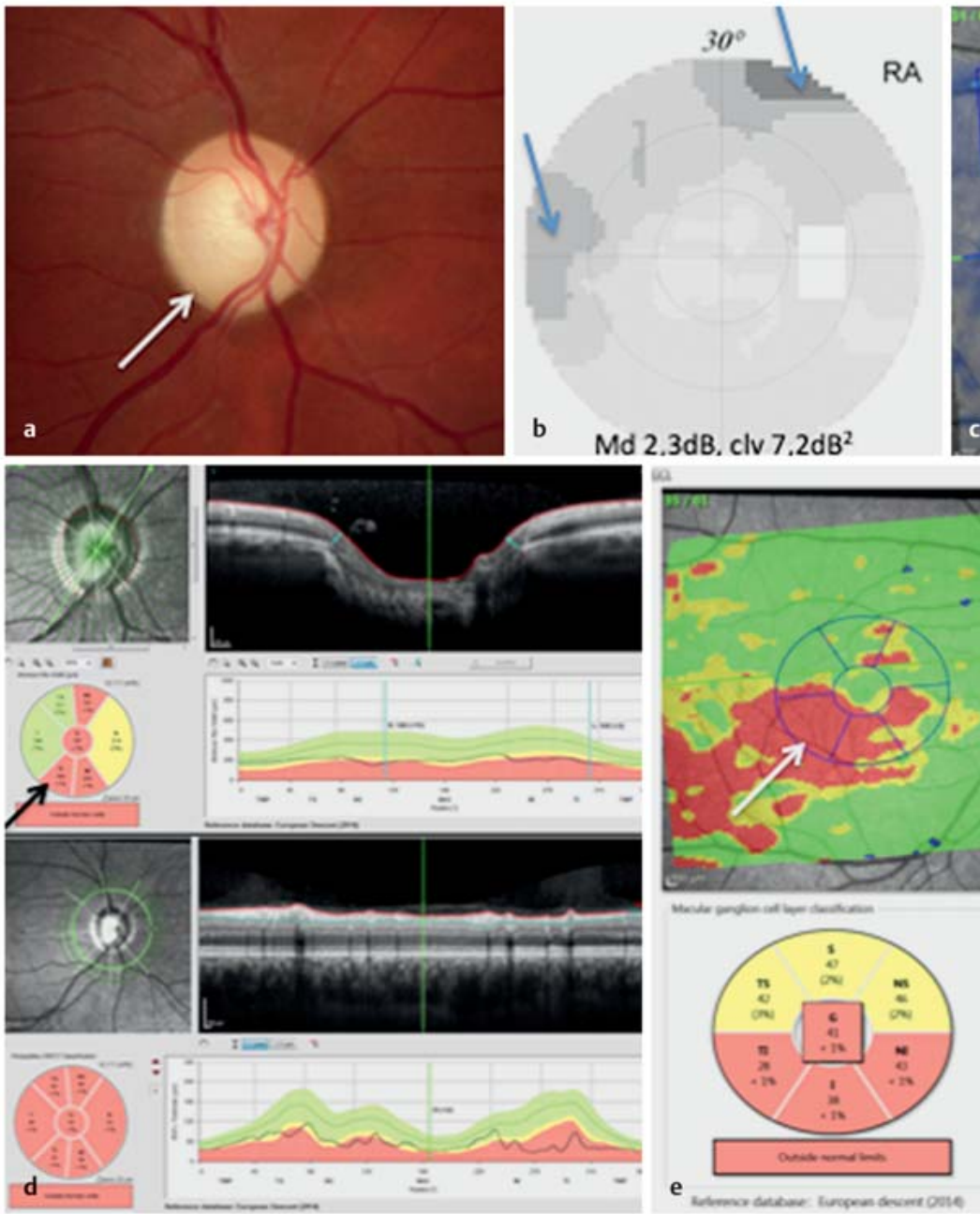
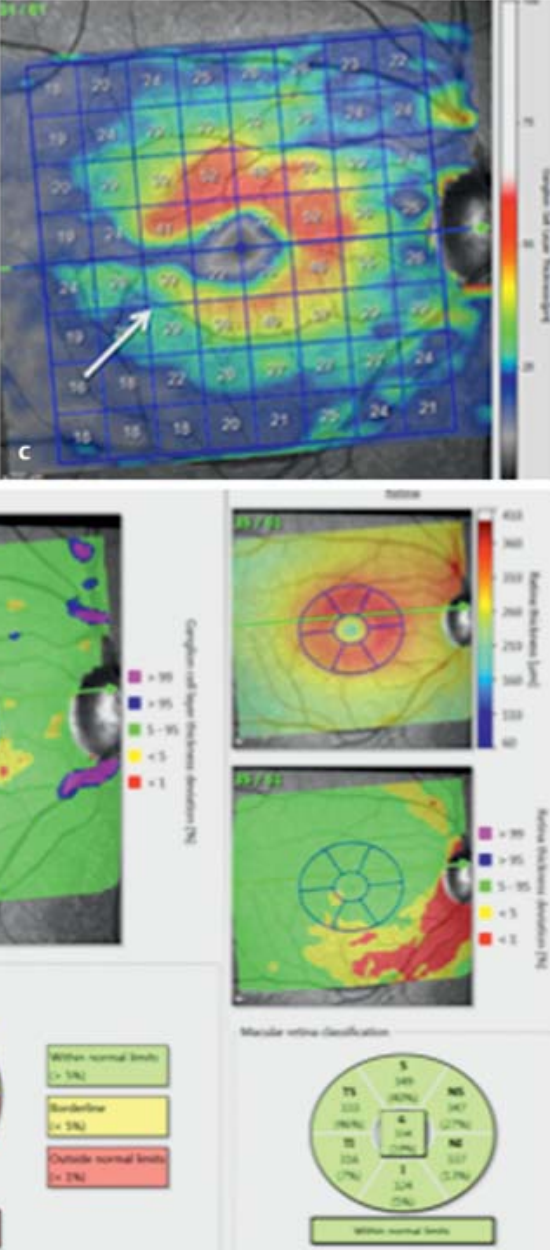

Abb. 2 Familiäres juveniles Offenwinkelglaukom. 
Exkavationsbeschaffenheit (hell), im Verhältnis zur NRRForm (dunkel) geben. Fokale RNFL-Defekte sind sehr gut detektierbar (dunkel auf hell).

Die zentrale Makulatopografiekarte sollte die perifoveolare Verdickung der Netzhaut als ringförmige Erhebung darstellen (Donut-Form). Verschiedene Hersteller verwenden farbkodierte Dickenkarten, auf denen Abweichungen von der Norm schnell erfasst werden können. Auch Abweichungskarten zur Normalpopulation werden angeboten, die in Grün, Gelb und Rot Auskunft über Abweichungen von Normschichtdicken geben. Auch sollten bei Auffälligkeiten der Topografie noch einmal das BScan-Bild und die Segmentierung überprüft werden.

Typischerweise atrophiert bei Glaukomen, korrespondierend zu den RNFL-Ausfällen temporal oben und unten, der temporale Anteil des GCL-Rings zuerst. In dieser Region liegen die Axonsomata. Die Trennlinie ist die Achse Fovea-Papillenmitte, die Raphe. Fortgeschrittene Glaukome zeigen nicht selten eine diffuse Verdünnung von retinaler Nervenfaserschicht (RNF), minimaler neuroretinaler Randsaumbreite (BMO-MRW) sowie Ganglienzellschicht (GCL) im OCT.

Die Asymmetrie rechts versus links ist ebenfalls ein bedeutsamer Marker für eine einseitig betonte Glaukomerkrankung. Auch hier werden zu diesem Zweck in den OCT-Programmen Symmetriedarstellungen angeboten. Die Asymmetrie zwischen oberer und unterer Makulaschichtdicke ist bei der GCL-Beurteilung wertvoll, da auch hier entweder oben oder unten die Atrophie mehr betont ist.

\section{TIPP}

Bevor OCT-Schichtdickenmesswerte im Vergleich zur Normpopulation für Therapieentscheidungen herangezogen werden, muss man sich über zahlreiche Einflussgrößen (Segmentierungsfehler, Sektorenzuordnung) im Klaren sein, um sich durch eine sogenannte „Green Disease“ (OCT normwertig, Patient aber erkrankt) oder durch eine „Red Disease“ (OCT pathologisch, Patient jedoch gesund) nicht täuschen zu lassen.
Im Zweifel, ob eine Glaukomerkrankung bereits zugrunde liegt, gibt die Verlaufsbeobachtung eine gute Information. Ist eine Schichtdickenabnahme (Ausgangswert der peripapillären Nervenfaserschichtdicke [normale, mittlere $\mathrm{RNFL}=$ ca. $100 \mu \mathrm{m}$ ) ausgeprägter (RNFL-Verlust $>1,2 \mu \mathrm{m} /$ Jahr) als der Altersverlust (RNFL ca. 0,6 $\mu \mathrm{m} /$ Jahr), so kann auch dies als eine fortschreitende Atrophie gewertet werden. Die grafische Darstellung als Ereignis oder Trend gibt hier Klarheit.

Die Trendanalyse zeigt eine schlechtere Ortsauflösung als die Ereignisanalyse, gibt dem Untersucher jedoch durch eine statistische Aussage (wieviel $\mu \mathrm{m}$ pro Jahr Verlust, mit welcher statistischen Signifikanz) etwas mehr Sicherheit in der Beurteilung einer Progression. Hierzu ist es ratsam, den Patienten öfter als jährlich, z.B. viertel- oder halbjährlich einzubestellen, damit kurzfristige Änderungen statistisch eher bemerkt werden. Da in der Trendanalyse erst nach 5 Messungen statistische Signifikanzen gezeigt werden, kann der Patient z. B. nach der Basisuntersuchung nach 3 Monaten und dann in den ersten 2 Jahren weitere 4-mal untersucht werden.

Ursachen für Artefakte und Fehlmessungen liegen einmal in der Aufnahmetechnik und zum zweiten in der individuellen Pathologie des untersuchten Auges. Letztere sind einerseits anatomische Besonderheiten wie vitreoretinale Traktionen, zystoide Ödeme (wie Verbreiterung der makulären inneren nukleären Schicht), weiche Drusen, myope Dehnungsareale, andererseits nicht glaukomassoziierte Optikuspathologien wie Drusen oder einfache Optikusatrophie mit aufsteigenden und absteigenden Ursachen. Aus diesem Grund ist es entscheidend, dass der Augenvorder- und -hinterabschnitt zur OCT-Aufnahme sorgfältig klinisch untersucht wurde. Das Gleiche gilt für die Musterung der OCT-B-Scans auf Segmentierungsfehler, anatomische Besonderheiten (Papillenrandblutungen) und Bildqualität.

Merke

Eine OCT-Untersuchung ist kein Diagnoseautomat vergleichbar einer Black Box. Dieses Thema wird mit der zunehmenden Anwendung Künstlicher Intelligenz in Zukunft noch bedeutsamer. 


\section{Worauf muss ich bei der OCT-Bildaufnahme achten?}

Die korrekte Positionierung des Patienten zur Vermeidung einer Verkippung (Tilt) des Kopfes hat einen großen Einfluss auf Verlaufsuntersuchungen, da diese nicht vollständig von dem Gerät korrigiert werden kann. Im Verlauf können unterschiedliche Kopfneigungen nach links oder rechts zu unterschiedlichen Lagen in der x,y-Position des Fundusbildes und somit zu rotatorischen Artefakten vor allem bei zirkumpapillären Kreisscans führen. Ein Beispiel für die Möglichkeit, dies in der Verlaufsuntersuchung auszugleichen, ist die Ausrichtung des Papillenzentrums auf die Fovea (FoDi, Heidelberg Engineering) für die korrekte Alignierung des peripapillären Kreisscans im Verlauf.

Vor allem dreidimensionale Aufnahmen erfordern multiple, gut alignierte B-Bilder ohne Bildversatz (z.B. durch APS = automated Positioning System). Hauptsächlich sind unwillkürliche Augenbewegungen der Grund für Bewegungsartefakte, die sich negativ auf die Bildqualität auswirken. Tremor, langsames Abweichen und Mikrosakkaden gilt es zu korrigieren. Die Hersteller versuchen, dies durch unterschiedliche Hard- und Softwarelösungen auszugleichen (z.B. TruTrack, Heidelberg Engineering; $V^{T r a c}$ Active Tracking, Optovue; FastTrac, Zeiss; SmartTrac, Topcon).

Das Gleiche gilt für die Wahl des korrekten Abstandes des Auges vom Objektiv, welche die Qualität und Position des Bildes (Sensitivity-Roll-off bei Spectral-Domain OCT) beeinflusst.

Einem trockenen Auge mit pathologischem Tränenfilm begegnet man mit der Gabe von künstlichen Tränen vor der Untersuchung. Eine lange Untersuchungsdauer kann zur Austrocknung der Korneaoberfläche führen und dadurch zu einer teils erheblichen Minderung der Bildqualität, da Tränenfilm und Hornhautepithel den ersten Übergang von Luft zum festen Medium darstellen und somit die Brechung des Laserstrahls beeinflussen. Hier kann mit der Anwendung von Tränenersatzmitteln vor der Bildaufnahme entgegengewirkt werden.

Die Fixation, sei es intern oder extern, kann bei schlechtem Visus oder funktioneller Einäugigkeit herausfordernd sein.

Die korrekte Ausleuchtung des Infrarot-Fundusbildes ohne Abschattungen am Rand sowie das Scharfstellen der zu untersuchenden Struktur sind essenziell.
Die korrekte Positionierung und Zentrierung des peripapillären Kreisscans sowie die Zentrierung des Makulavolumens auf die Foveola sind entscheidend für den korrekten Vergleich mit der Normdatenbank.

Hohen Astigmatismen sowie hohen Achslängen kann begegnet werden durch folgende Maßnahmen:

- Tragen von Kontaktlinsen,

- Vorsatz astigmatischer Gläser und

- richtige Gerätevoreinstellung:

- normal,

- myop,

- hochmyop.

TAKE HOME MESSAGE

Die Bildqualität muss im Normbereich sein, damit die Untersuchung aussagekräftig wird.

\section{Was sind glaukomassoziierte Einflussgrößen bei der OCT-Messung?}

\section{FALLBEISPIEL 2}

Männlicher Patient, 67 Jahre alt, Normaldruckglaukom. Am linken Auge besteht eine NRR-Kerbe der Papille bei 5 Uhr (a, weißer Pfeil), temporal zeigt die retinale Nervenfaserschicht (RNFL) im OCT eine deutliche Verdünnung (b, schwarzer Pfeil) ( $\bullet$ Abb. 3 ). Die Netzhautdicke (c, schwarzer Pfeil) ist oben und unten symmetrisch, obwohl auf dem Infrarotbild (d, weißer Pfeil) ein deutlicher RNFL-Verlust unten bogenförmig sichtbar ist (f, weißer Pfeil). In diesem Areal (d, grüne Linie) ist die innere Körnerschicht deutlich zystoid aufgetrieben (e, h, weißer Pfeil), die Ganglienzellschicht (GCL) dagegen verdünnt (g, weißer Pfeil). Insgesamt zeigt sich damit, netto, keine Verdünnung der Netzhautgesamtdicke.

Auf der unten (i) dargestellten Abweichungskarte sieht man deshalb auf der GCL-Dickenkarte in Rot eine deutlich ausgeprägtere Atrophie (Mitte, rot) als in der Netzhautdickenkarte (rechts oben). 


\section{FALLBEISPIEL 2 (FORTSETZUNG)}
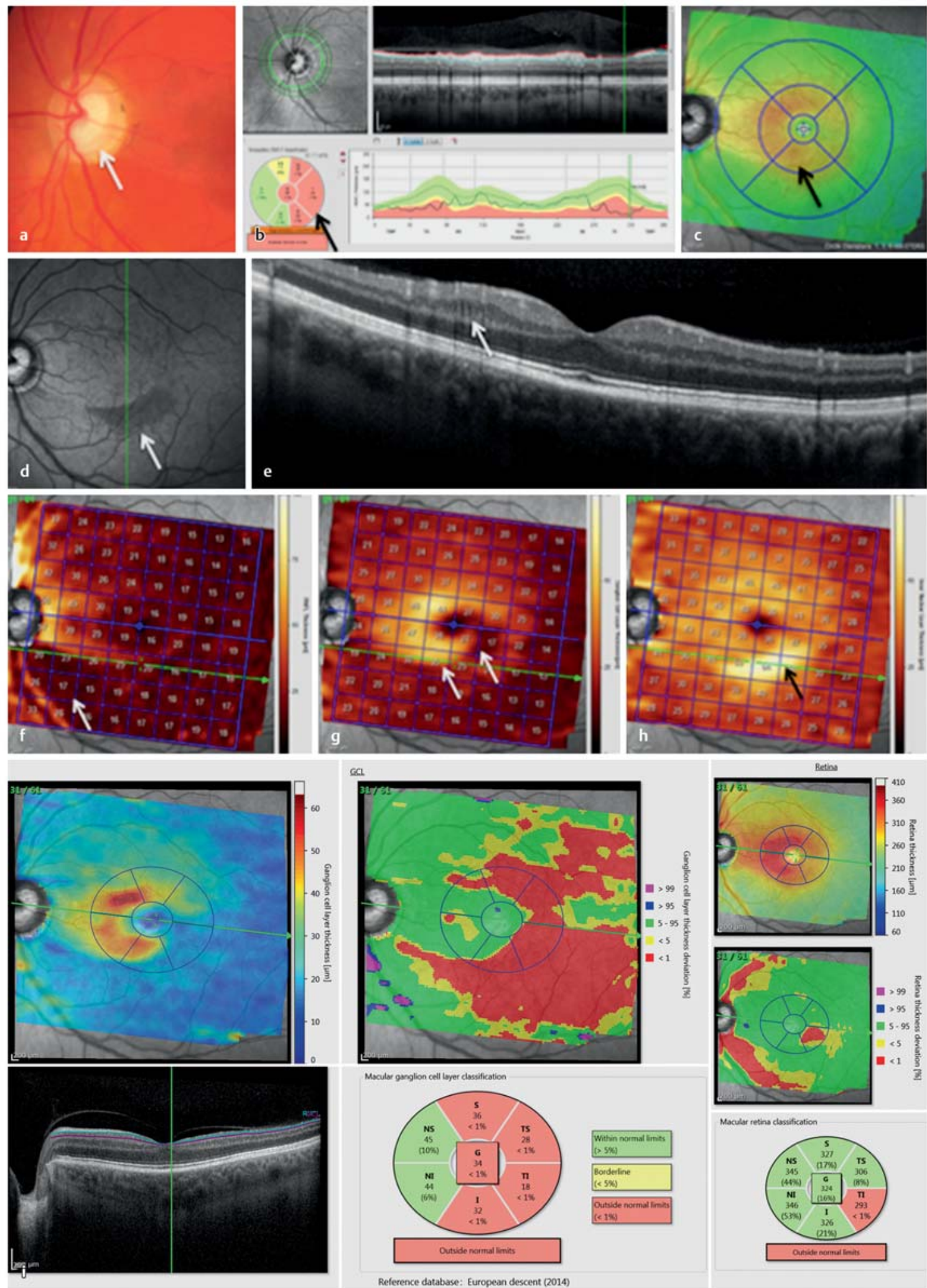

- Abb. 3 Normaldruckglaukom. 
Bei fokalen Defekten der RNFL kann es infolge des fokalen Verlustes der korrespondierenden Ganglienzellschicht zu einer zystoiden Erweiterung der darunter liegenden inneren Körnerschicht kommen. Die Aufweitung der inneren Körnerschicht und die darüber liegende Verdünnung der Ganglienzellschicht (GCL) kann netto zu einer pseudonormalen Gesamtnetzhautdicke führen, welche unauffällig erscheint, obwohl bereits ein deutlicher Schaden vorhanden ist. Die zystoide Erweiterung kann hinweisend sein auf eine Progression des Glaukoms. Diese Veränderung, die sehr gut in B-Scans des Makulavolumens gesehen werden kann, ist ebenfalls bei Multipler Sklerose und anderen Optikusatrophien ein Marker für den Schweregrad der Erkrankung.

Ab einer mittleren RNFL-Schichtdicke von ca. $50 \mu \mathrm{m}$ ist eine Progression nicht weiter zu erwarten [7]. In diesem Stadium erscheinen die makuläre GCL-Dickenmessung und Reduktion der Kapillardichte in der OCT-Angiografie aussagekräftiger [8].
Papillenrandblutungen in der RNFL, die sich in die Peripherie ausdehnen, können zu einer artifiziellen Verdickung der Schichtmessung mit einer nachfolgenden Verdünnung der RNFL führen.

Um glaukomassoziierte anatomische Veränderungen beurteilen zu können, sollten die hochauflösenden B-Scans, wie histologische Schnitte betrachtet werden.

\section{Was sind nicht glaukomassoziierte anatomische Einflussgrößen bei der OCT-Messung?}

Von der Glaukomerkrankung unabhängige Veränderungen können die Anatomie und damit die Messung der Dicke der retinale Nervenfaserschicht (RNFL) und der Ganglienzellschicht (GCL) und der minimalen neuroretinalen Randsaumbreite (BMO-MRW) beeinflussen. Am häufigs-

\section{FALLBEISPIEL 3}

52-jährige Patientin mit Glaukomverdacht bei schräger, vitaler, myopischer Papille (Bild links oben) ( $>$ Abb. 4).

Das Makula-OCT (rechts) zeigt eine unregelmäßige Topografie der Netzhautdicke durch Segmentierung der epiretinalen Membran (unten, B-Bild), die ebenfalls zu einer Verdickung der zentralen Netzhaut führt.

Damit ist diese Untersuchung nicht aussagekräftig.
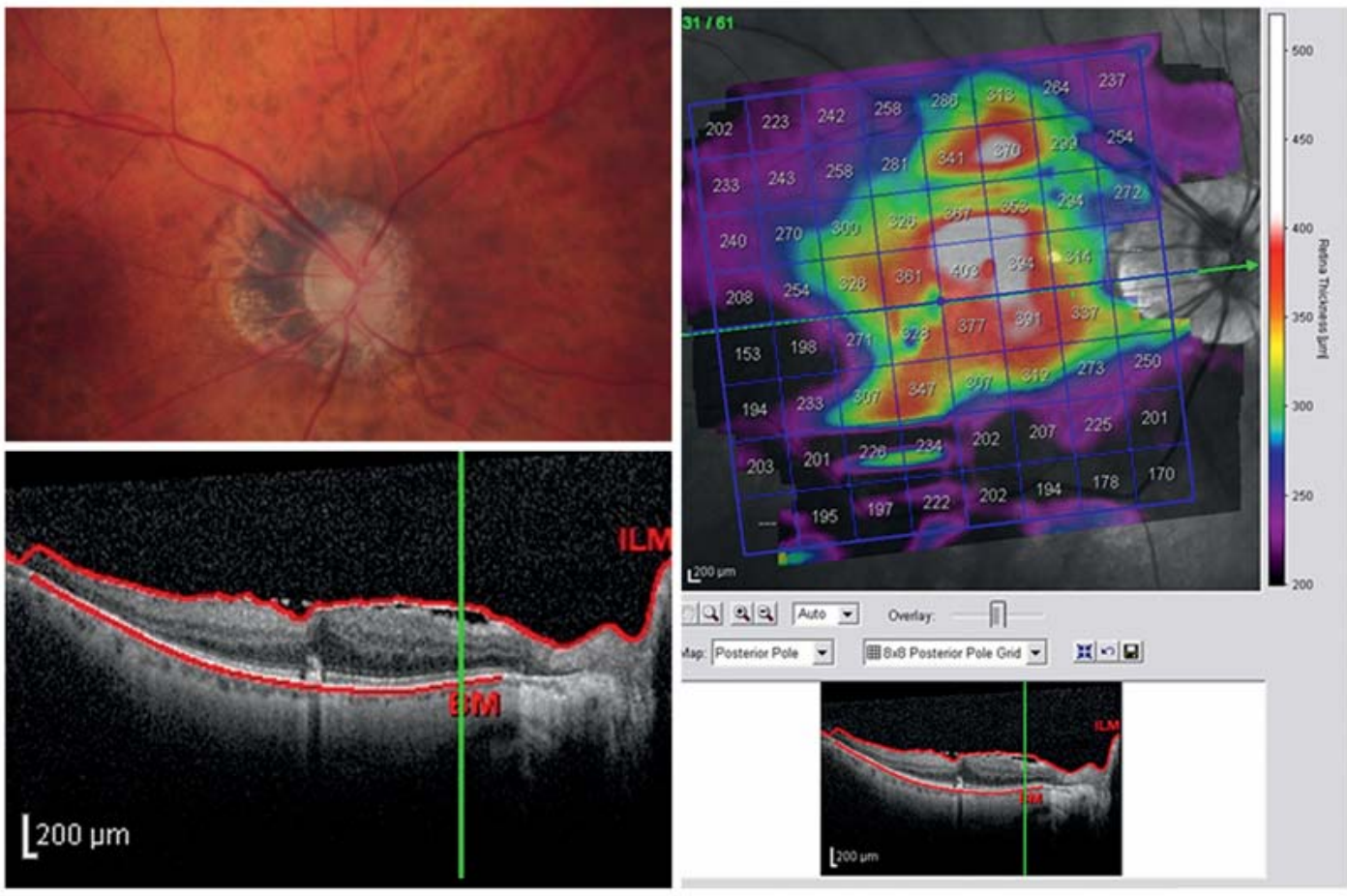

- Abb. 4 Glaukomverdacht. 
ten sind dies Veränderungen des vitreoretinalen Interface wie epiretinale Membranen und Traktionen, die zur Aufspleißung vor allem der bereits erkrankten RNFL führen. Auch durch die Traktion der zentralen Makula können Volumenscans der Makula falsch segmentiert werden.

Intraretinal sind es meist zystoide Ödeme bei neovaskulärer altersassoziierter Makuladegeneration (AMD), diabetischer Retinopathie oder retinalen Gefäßverschlüssen, die eine korrekte Segmentierung der Netzhautschichten verhindern oder zu hohe Werte für die Messung verursachen. Eine altersabhängige Makuladegeneration mit weichen, konfluierenden Drusen und Abhebungen der retinalen Pigmentepithelschicht können auch die Segmentierung der RNFL im OCT und damit die Schichtdickenmessung beeinträchtigen.

Problematisch sind artifizielle Messungen von retinalen Gefäßen oder Abschattungen, Glaskörperverdichtungen an der Membrana limitans interna oder untypische Endigungen der Bruch-Membran. Letztere sind temporal bei flach auslaufenden, myopischen Papillen sehr peripher zu finden und führen zu ungewollten Dickenmessungen äußerer Netzhautschichten. Aus diesem Grund müssen alle Messpunkte im B-Scan vom Untersucher kontrolliert und bei Bedarf korrigiert werden.

Hochmyope Augen mit einer hohen Achsenlänge und posterioren Staphylomen zeigen eine unterschiedliche Verteilung der retinalen Nervenfasern (RNF) am Fundus und damit auch am Scankreis. Hier neigen die RNFL-Gipfel dazu, sich nach temporal zu verlagern, und fallen deshalb außerhalb der normalen TSNIT-Grafik. Dieses Artefakt versuchen die OCT-Geräte mit der Berücksichtigung der Hornhautkrümmung und Refraktion auszugleichen, deren Eingabe in der Praxis nicht immer geschieht, sodass von der Software Standardwerte verwendet werden. Der Scankreis, der unter einem festen Winkel eingestrahlt wird, wird durch die hohe Achslänge des hochmyopen Auges zu weit von der Papille entfernt abgebildet. Damit verschieben sich die RNF-Maxima, bedingt durch den bogenförmigen Verlauf der RNF zur Papille zu weit nach temporal.

Auch posteriore Staphylome und schräge Sehnerveneintritte machen teilweise eine sinnvolle Segmentierung der RNF unmöglich. Das SPECTRALIS-OCT (Heidelberg Engineering, Heidelberg) bietet hier Scankreise mit einem höheren Durchmesser als 3,5 mm an, um dann in Regionen außerhalb des Staphyloms eine RNFL-Messung durchzuführen (4,1 und 4,7 mm). Alternativ kann die makuläre Ganglienzellschichtdicke als Glaukommarker analysiert werden. Gelingt dies nicht, müssen andere, glaukomrelevante Variablen, wie Papillenmorphologie oder fokale RNF-Defekte, betrachtet werden.
Papillenform und Papillengröße können die Analyse der minimalen neuroretinalen Randsaumbreite im OCT beeinflussen. Sehr kleine Papillen bewirken aufgrund des geringen Durchmessers des Optikoskleralkanals Werte der minimalen neuroretinalen Randsaumbreite (BMO-MRW) im oberen Perzentilenbereich. Große Papillen zeigen hingegen Werte am unteren Perzentilenbereich bedingt durch die NRR-Verteilung entlang eines größeren Skleralkanalumfangs.

Vor allem bei myopischen Augen ist die Papillenfläche und BMO-Fläche mit der Achsenlänge positiv korreliert [9]. Dies kann zu einer Fehleinschätzung der BMO-MRW führen. Große und kleine Bruch-Membran-Öffnungen beeinflussen den Grad der Abweichung von der Normalpopulation [6]. Bei frühen Glaukomen kann BMO-MRW bei kleinen Papillen eine größere Normabweichung zeigen als die RNFL. Bei großen Papillen verhält es sich eher umgekehrt.

TAKE HOME MESSAGE

Alle anatomischen, pathologischen Veränderungen des Fundus können OCT-Messungen beeinflussen.

Wie unterscheide ich andere Ganglienzellund Optikusatrophien in der Differenzialdiagnose zu Glaukomen in der OCT?

Auch wenn Aufnahme und Segmentierung von neuroretinalem Randsaum (NRR), retinaler Nervenfaserschicht (RNFL) und Ganglienzellschicht (GCL) fehlerfrei gelingen, gibt es dennoch pathologische Befunde, die nicht mit einer Glaukomerkrankung zusammenhängen. Alle Ursachen einer aufsteigenden (von den inneren Netzhautschichten bis zur Lamina cribrosa) und einer absteigenden (von der Lamina cribrosa bis zum Ganglion geniculatum) Optikusatrophie beeinflussen OCT-Schichtdickenmessungen und können im pathologischen Bereich liegen.

\footnotetext{
Cave

Prinzipiell muss bei einem Zweifel an einem Glaukomschaden die Papille ophthalmoskopiert und eine einfache Optikusatrophie ausgeschlossen werden. Unterstützend wird eine Gesichtsfelduntersuchung durchgeführt.
}

Aber auch bestimmte OCT-Messwertkonstellationen können auf eine nicht glaukomassoziierte Atrophie hinweisen. 


\section{FALLBEISPIEL}

Fall 4

Männlicher Patient, 42 Jahre alt. Seit einigen Tagen auf 0,15 geminderter Visus im rechten Auge; afferente Pupillendifferenz positiv. Der Patient wurde aufgrund von Gesichtsfeldausfällen mit Glaukomverdacht vorgestellt.

Betrachtet man nur den OCT-Befund, so fällt dem Untersucher eine grenzwertige minimale neuroretinale Randsaumbreite (BMO-MRW; a) bei deutlich reduzierter retinaler Nervenfaserschicht (RNFL) (b) und Netzhautatrophie (c) auf ( $\bullet$ Abb. 5). Die Ophthalmoskopie erklärt den Widerspruch: Es handelt sich nicht um ein Glaukom, sondern um eine einfache, absteigende Optikusatrophie mit temporaler Blässe (d) nach Neuritis nervi optici.
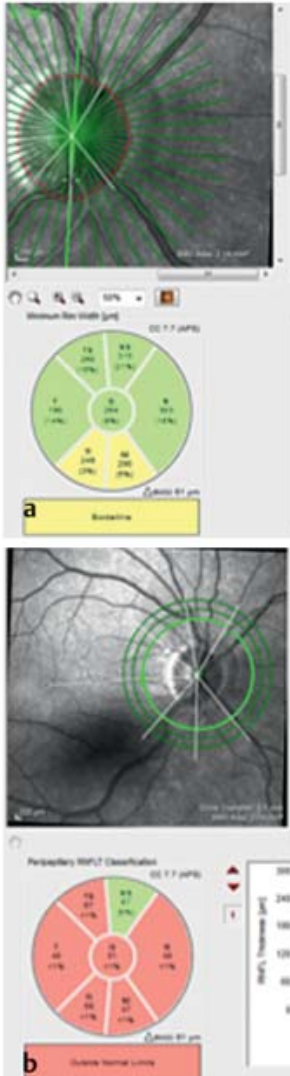

b
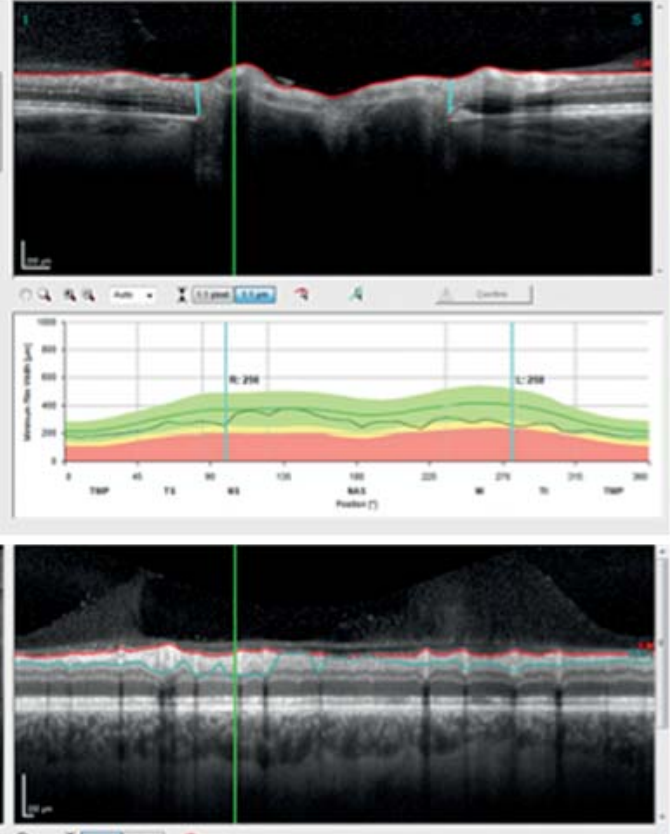

( $\mathrm{x}$ and 3

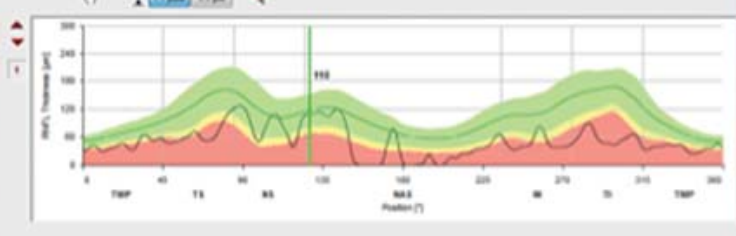

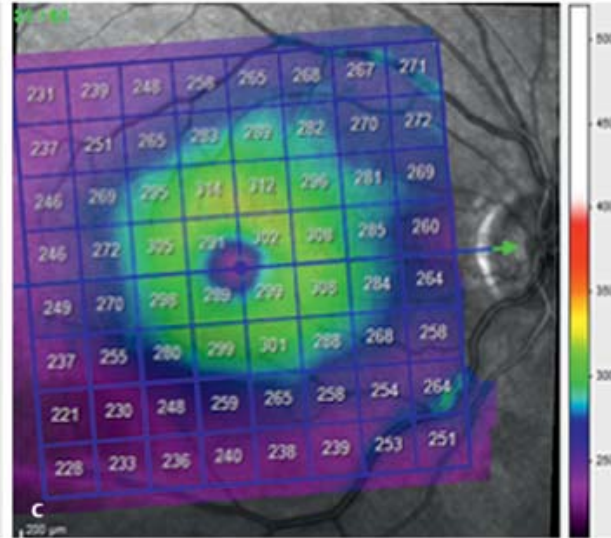

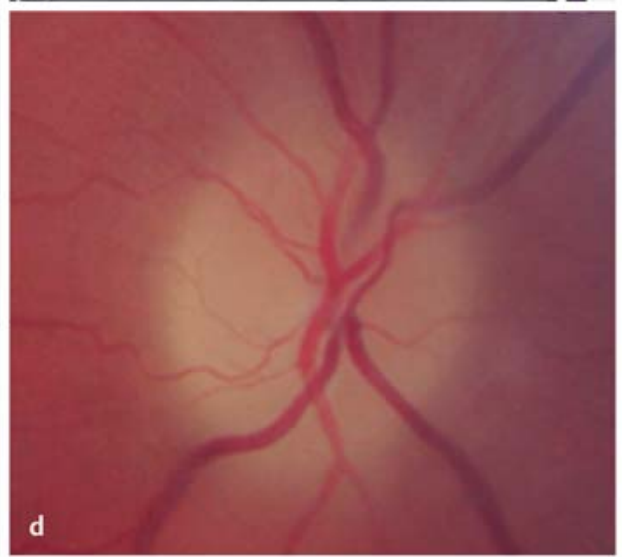

- Abb. 5 Differenzialdiagnose bei Glaukomverdacht.

Merke

Prinzipiell sind bei Glaukomen Dickenreduzierungen der retinalen Nervenfaserschicht (RNFL) und der minimalen neuroretinalen Randsaumbreite (BMOMRW) bei regulärer Papillenform temporal oben und unten am ausgeprägtesten und temporal lange weniger reduziert.

Abweichungen von diesem Muster bei regulärer Papillenform mit normaler Position des retinalen Gefäßbaums (meist nasal oben) wie bei einer temporal betonten RNFL-Dickenabnahme weisen z.B. auf postneuritische
Atrophien hin. Eine segmental, horizontal ausgeprägte Reduktion der RNFL-Dicke kann mit einem retinalen, hemisphärischen Gefäßverschluss oder einer nicht arteriitischen Papillenatrophie zusammenhängen. Bei der einfachen Optikusatrophie kommt es zu einer Abflachung des neuroretinalen Randsaums (Erhalt von Astroglia und Verlust von Axonen) bei nur gering vergrößerter Exkavation, weshalb die NRR-Dicke seltener auffällig ist oder weniger schichtdickenreduziert ist.

Der makuläre Ganglienzellschichtdickenverlust bei Glaukomen folgt dem bogenförmigen, fokalen RNFL-Ausfall 
mit Atrophie der Ganglienzellsomata. Somit ist die Schichtdickenkarte temporal oben oder temporal unten zu Beginn der Erkrankung pathologisch (Form eines angebissenen Donuts oder Form eines Nautilusgehäuses). Bei einer kompletten, einfachen Optikusatrophie ist die GCL-Dicke diffus reduziert und ähnelt einer weit fortgeschrittenen, glaukomatösen Optikusatrophie. Gleichzeitig ist die RNFL-Dicke diffus ausgeprägt pathologisch. Im Kontrast zu diesen Befunden steht meistens nur eine relativ gering verdünnte BMO-MRW-Dicke in der OCT.

\section{TIPP}

Sind die makuläre Dicke der Ganglienzellschicht und die retinale Nervenfaserschicht in der OCT reduziert und die minimale Randsaumbreite fast unauffällig, sollte an eine neurologische, degenerierende Erkrankung wie Morbus Alzheimer gedacht werden. Erstaunlicherweise findet man nicht selten erste pathologische OCT-Veränderungen im Bereich der Ganglienzellschichtdicke.

Eine vertikale Ausrichtung des Verdünnungsmusters der Ganglienzellschicht homonym oder binasal weist auf eine absteigende Atrophie des N. opticus bei zerebralen Mittellinienerkrankungen wie einem kompressiv wachsenden Hypophysenadenom oder auf eine links- oder rechtshirnige Erkrankung mit transsynaptischer Degeneration hin. Der OCT-Untersuchung sollte dann eine Gesichtsfelduntersuchung nachfolgen, da hier nicht selten zum OCT-Befund kongruente, homonyme oder bitemporale Ausfälle offenbar werden.

\section{Cave}

Die Differenzialdiagnose der glaukomatösen Papillenschädigung kann für den Patienten auch von vitaler Bedeutung sein.

\section{Was tun bei Papillendrusen, Papillenödem, Papilla leporina?}

Papillendrusen und Papillenödem führen zu einer überhöhten minimalen Randsaumbreitenmessung. Jedoch erscheint die retinale Nervenfaserschicht (RNFL) bei Ödemen durch Aufstau, Entzündung und Ischämie noch dicker und neigt bei einer Stauung zur Faltenbildung (Patton-Ringe). Abschattungen durch retinale Gefäße, Ödem und Drusen können die Darstellung der BruchMembran-Endigung und damit die Segmentierung erschweren. Damit gelingt die Segmentierung nicht mehr.

Die Dicke der retinalen Nervenfaserschicht (RNFL) ist eine alternative Messgröße zur Beurteilung einer Atrophie, welche sowohl bei Drusen als auch nach einem neuritischen/ischämischen Ereignis eintritt und eine Verdün- nung der RNFL zeigt. Auch die Messung der verdünnten Ganglienzellschicht (GCL) ist ein guter Marker zur Beurteilung einer nach dem Ereignis eintretenden einfachen Atrophie.

Eine komplexe Papillenatrophie führt durch Vermehrung von Astroglia und gleichzeitigem Verlust von Axonen (z. B. nach arteriitischer Papillenapoplexie) zu einer blassen, randunscharfen Papille. Dies schlägt sich in einer reduzierten RNFL-Dicke im OCT-Bild bei übernormaler minimaler neuroretinaler Randsaumbreite (BMO-MRW) nieder. Auch hier ist die Beurteilung der verdünnten GCL zur Diagnosefindung hilfreich.

Myelinisierung der retinalen Nervenfaserschicht bei einer Papilla leporina führt ebenfalls zu einer artifiziellen Verdickung der BMO-MRW und RNFL-Dicke. Beides erschwert die Beurteilung eines Glaukomschadens. Auch hier empfiehlt es sich, auf die GCL-Dicke auszuweichen.

\section{KERNAUSSAGEN}

- Hochauflösende OCT-Technik erlaubt die Darstellung und Vermessung von retinaler Ganglienzellschicht (GCL) und Nervenfaserschicht mit hoher Auflösung ähnlich einem histologischen Schnitt.

- Die Messung glaukomrelevanter Strukturen am Augenhintergrund mit dem OCT erleichtert die Diagnosestellung und Verlaufsbeobachtung bei frühen Glaukomen.

- Die farbkodierte Darstellung von Abweichungen von der Normalpopulation erlauben dem Untersucher einen schnellen Überblick auf pathologische Messungen.

- Die Bildqualität von OCT-Aufnahmen ist für die Zuverlässigkeit der Messungen entscheidend.

- Es ist die Aufgabe des befundenden Arztes, Artefakte und Fehlmessungen zu erkennen, um Fehlinterpretationen zu vermeiden.

- Artefakte können sowohl durch glaukomassoziierte Pathologien als auch durch nicht glaukomatöse Veränderungen am hinteren Augenpol verursacht werden.

- Myopische Papillen mit verdünnten Netzhautschichten und schrägem Sehnerveneintritt sind auch für die OCT-Untersuchung eine Herausforderung.

- Bedeutsam ist in der Differenzialdiagnose das Erkennen neurologischer Erkrankungen und ihren Veränderungen in der retinalen Ganglienzell- und Nervenfaserschicht. 
Claudia Holzhey und Martin Long von der Heidelberg Engineering Academy gilt besonderer Dank für den jahrelangen, intensiven Austausch zu OCT-Aufnahmen und die kritische Durchsicht des Artikels.

Interessenkonflikt

Erklärung zu finanziellen Interessen

Forschungsförderung erhalten: nein; Honorar/geldwerten Vorteil für Referententätigkeit erhalten: ja, von einer anderen Institution (Pharma- oder Medizintechnikfirma usw.).; Bezahlter Berater/interner Schulungsreferent/Gehaltsempfänger: nein; Patent/Geschäftsanteile/Aktien (Autor/Partner, Ehepartner, Kinder) an Firma (Nicht-Sponsor der Veranstaltung): nein; Patent/Geschäftsanteile/Aktien (Autor/Partner, Ehepartner, Kinder) an Firma (Sponsor der Veranstaltung): nein.

Erklärung zu nichtfinanziellen Interessen

Mitgliedschaften: ARVO, DOG, BVA, NOS, SIDUO

Autorinnen/Autoren

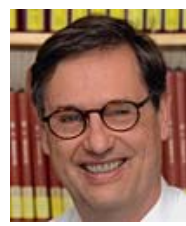

\section{Christian Y. Mardin}

Prof. Dr. med. Medizinstudium 1985-1991 (Friedrich-Alexander-Universität ErlangenNürnberg), Promotion 1991. Facharztausbildung 1991-1996 an der Universitätsaugenklinik Erlangen); Außerplanmäßiger Professor 2006. Tätig als leitender Oberarzt der Universitätsaugenklinik Erlangen seit 2013. Mitglied in DOG, ARVO, SIDUO, NOS. Außerklinisches Engagement für die Christoffel Blindenmission in Nepal seit 2002 und Deutsch-Vietnamesische Zusammenarbeit in der Augenheilkunde.

Korrespondenzadresse

Prof. Dr. Christian Y. Mardin

Universitätsklinikum Erlangen

Augenklinik

Schwabachanlage 6

91054 Erlangen

christian.mardin@uk-erlangen.de

Wissenschaftlich verantwortlich gemäß Zertifizierungsbestimmungen

Wissenschaftlich verantwortlich gemäß Zertifizierungsbestimmungen für diesen Beitrag ist Prof. Dr. Christian Y. Mardin, Erlangen.
Literatur

[1] Schrems-HösI L, Schrems W, Lämmer R et al. Precision of optic nerve head and retinal nerve fibre layer parameter measurements by spectral-domain optical coherence tomography. J Glaucoma 2018; 27: 407-414

[2] Mardin C. Structural diagnostics of course observation for glaucoma. Ophthalmologe 2013; 110: 136-144

[3] Oddone F, Lucenteforte E, Michelessi M et al. Macular versus nerve fibre layer parameters for diagnosing mainfest glaucoma. Ophthalmology 2016; 123: 939-949

[4] Chauhan BC, O'Leary N, AlMobarak FA et al. Enhanced detection of open-angle glaucoma with an anatomically accurate OCT-derived NRR parameter. Ophthalmology 2013; 120: 535-543

[5] Pollet-Villard F, Chiquet C, Romanet JP et al. Structure-function relationships with spectral-domain optical coherence tomography retinal nerve fiber layer and optic nerve head measurements. Invest Ophthalmol Vis Sci 2014; 55: 2953-2962

[6] Gmeiner JM, Schrems WA, Mardin CY et al. Comparison of Bruch's membrane opening minimum rim width and peripapillary retinal nerve fibre layer thickness in early glaucoma assessment. Invest Ophthalmol Vis Sci 2016; 57: 575-584

[7] Mwanza JC, Kim HY, Budenz DL et al. Residual and dynamic range of retinal nerve fiber layer thickness in glaucoma: comparison of three OCT platforms. Invest Ophthalmol Vis Sci 2015; 56: 6344-6351

[8] Moghimi S, Bowd C, Zangwill LM et al. measurement floors and dynamic ranges of OCT and OCT angiography in glaucoma. Ophthalmology 2019; 126: 980-988

[9] Tan NYQ, Sng CCA, Jonas JB et al. Glaucoma in myopia: diagnostic dilemmas. Br J Ophthalmol 2019; 103: 1347-1355

\section{Bibliografie}

DOI https://doi.org/10.1055/a-1098-3466

Online-publiziert 3.2.2020 | Klin Monatsbl Augenheilkd 2020; 237: $539-551$

(c) Georg Thieme Verlag KG Stuttgart · New York

ISSN 0023-2165 


\section{Punkte sammeln auf CME.thieme.de}

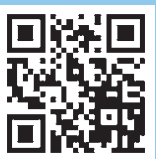

Diese Fortbildungseinheit ist in der Regel 12 Monate online für die Teilnahme verfügbar.

Den genauen Einsendeschluss finden Sie unter https://cme.thieme.de.

Sollten Sie Fragen zur Online-Teilnahme haben, finden Sie unter https://cme.thieme.de/hilfe

eine ausführliche Anleitung. Wir wünschen viel Erfolg beim Beantworten

der Fragen!

Unter https://eref.thieme.de/CXD68B] oder über den QR-Code kommen Sie direkt zur Startseite des Wissenstests.

VNR 2760512020158723235

\section{Frage 1}

Welche digitale axiale Auflösung bietet die Spectral-Domain OCT?
A $10 \mu \mathrm{m}$
B $50 \mu \mathrm{m}$
C $0,2 \mathrm{~mm}$
D $3,9 \mu \mathrm{m}$
E $860 \mathrm{~nm}$

\section{Frage 2}

Die mittlere peripapilläre Nervenfaserschichtdicke in der OCTStandardmessung beträgt am ehesten ...
A $100 \mu \mathrm{m}$.
B $69 \mu \mathrm{m}$.
C $256 \mu \mathrm{m}$.
D $30 \mu \mathrm{m}$.
E $50 \mu \mathrm{m}$.

\section{Frage 3}

Einen der folgenden Befunde zeigt das Infrarot-Reflexionsbild der OCT-Aufnahme nicht gut. Welchen?

A fokale Defekte der retinalen Nervenfaserschicht

B Papillenrandblutungen

$C$ retinale Gefäße

D Exkavation

E einen neuroretinalen Randsaum

\section{Frage 4}

Wo zeigt sich die erste Verdünnung von retinaler Nervenfaserschicht und neuroretinalem Randsaum (MRW-BMO) in der OCT-Messung?
A nasal
B temporal
C immer diffus über $360^{\circ}$
D temporal unten
E nasal oben

\section{Frage 5}

Wie groß ist der mittlere jährliche Altersverlust der peripapillären, retinalen Nervenfaserschicht einer Normalperson in der OCT-Messung?
A $1,5 \mu \mathrm{m}$
B $1,2 \mu \mathrm{m}$
C $0,1 \mu \mathrm{m}$
D $2,3 \mu \mathrm{m}$
E $0,6 \mu \mathrm{m}$

\section{Frage 6}

Glaukomassoziierte Messartefakte in der OCT-Aufnahme werden wodurch verursacht?
A zystoide Verbreiterung der makulären inneren, nukleären Schicht
B Verlust der myoiden Zone der Photorezeptoren
C epiretinale Membranen
D Drusen
E harte Exsudate

\section{Frage 7}

Nicht glaukomassoziierte Gewebsartefakte führen zu einer Verdickung der retinalen Nervenfaserschichtsegmentierung bei welchem der genannten Befunde?
A bei geografischer Atrophie des RPE
B bei epiretinaler Gliose
C bei Papillenkolobom
D bei myopischem Staphyloma posticum
$\mathrm{E}$ bei Papillenrandblutungen

\section{- Weitere Fragen auf der folgenden Seite...}




\section{Punkte sammeln auf CME.thieme.de}

\section{Fortsetzung...}

\section{Frage 8}

Hochmyope Augen zeigen in der OCT-Messung welche der folgenden Veränderungen?

A Verlagerung der Schichtmaxima der retinalen Nervenfasern (RNF) nach nasal

B Zone Gamma mit Bruch-Membran

C Verlagerung der RNF-Schichtmaxima nach temporal

D Mikropapillen

E verdickte minimale neuroretinale Randsaumbreite (BMO-MRW)

\section{Frage 9}

Neurologische Erkrankungen des Zentralnervensystems führen in der OCT-Darstellung des hinteren Pols nicht zu ...

A einer diffusen Verdünnung der Ganglienzellschicht.

B homonymen Atrophie der Ganglienzellschicht.

$C$ einem ausgeprägteren Verlust von retinalen Nervenfasern (RNF) als bei der minimalen neuroretinalen Randsaumbreite (BMO-MRW).

D dem typischen Verlust von RNF-Schichtdicke temporal oben.

E frühem Verlust der Ganglienzellschicht.

\section{Frage 10}

Papillendrusen und Papillenödem zeigen in der OCT-Untersuchung welchen der im Folgenden genannten Befunde?

A im Verlauf eine Verdünnung der Schichtdicke retinaler Nervenfasern

B von Beginn an eine verdünnte minimale neuroretinale Randsaumbreite (BMO-MRW)

C immer den gleichen, parallelen Verlauf von BMO-MRW und RNF

D keine Artefakte in der Segmentierung der Endigung der Bruch-Membran

E immer eine klare Unterscheidung voneinander 